\title{
Electromyographic pattern analysis and classification for a robotic prosthetic arm
}

doi:10.1533/abbi.2005.0039

\author{
M. José H. Erazo Macias ${ }^{1}$ and S. Alejandro Vega ${ }^{2}$ \\ ${ }^{I}$ Department of Electric and Electronic Engineering, Technological Institute of Reynosa, Reynosa Tamaulipas, México \\ ${ }^{2}$ Technological Institute of Superior Studies of Monterrey, Campus Queretaro, Querétaro, México
}

\begin{abstract}
This paper deals with the statistical analysis and pattern classification of electromyographic signals from the biceps of a person with amputation below the humerus. Such signals collected from an amputation simulator are synergistically generated to produce discrete elbow movements. The purpose of this study is to utilise these signals to control an electrically driven prosthetic or orthotic elbow with minimum extra mental effort on the part of the subject. The results show very good separability of classes of movements when a learning pattern classification scheme is used, and a superposition of any composite motion to the three basic primitive motions - humeral rotation in and out, flexion and extension, and pronation and supination. Since no synergy was detected for the wrist movement, different inputs have to be provided for a grip. In addition, the method described is not limited by the location of the electrodes. For amputees with shorter stumps, synergistic signals could be obtained from the shoulder muscles. However, the presentation in this paper is limited to biceps signal classification only.
\end{abstract}

Key words: Electromyographic, amputated, orthotic, pronation, supination.

\section{INTRODUCTION}

Since World War II, many attempts have been made to use external power to operate artificial prosthetic or orthotic arms (Alderson 1954). Electromyographic (EMG) signals from the body's intact musculature have been suggested and utilised by many researchers as an effective noninvasive method to provide commands to control an electrically powered artificial limb (Graupe et al. 1978; Lyman et al. 1974; Saidis and Stephenou 1977). Most of this research has produced single motion controls of the bang-bang type, which require special training and effort on the part of the subject.

The introduction of microcomputers has made it possible to experiment with more sophisticated signal detection and motion control of human prostheses. Graupe and Salahi (1978) and Graupe et al. (1978) proposed and successfully implemented a time series identification to recover control information from synergistic EMG signals

Corresponding Author:

M. José H. Erazo Macias

Department of Electric and Electronic Engineering

Technological Institute of Reynosa

Reynosa Tamaulipas

México

Email: erazo_joseh@mexico.com produced by the subject's intact musculature. However, this project was terminated before any conclusive design procedures were developed.

This paper presents an approach to detect, analyse, and classify synergistic EMG signals generated by the biceps of an above-the-elbow amputee in an attempt to move the artificial limb without extra mental effort. The method is by no means limited to this location of the electrodes (Graupe and Salahi 1979). However, since stronger synergies are expected from the arm muscles, they were prepared in this study. An elbow of three degrees of freedom is considered. It was built using a newly designed product (made in the Department of Electric and Electronic Engineering, Technological Institute of Reynosa, Tamaulipas, Mexico), while the mechanism and all its parts were made in the Technological Institute of Reynosa (Figure 1). The elbow could be driven to perform all possible simultaneous combinations in the following six primitive motions:

- Humeral rotation inside

- Humeral rotation outside

- Elbow flexion

- Elbow extension

- Elbow pronation of wrist

- Elbow supination of wrist

The wrist and hand grasp was not included in the final classification because no synergistic signals were obtained 


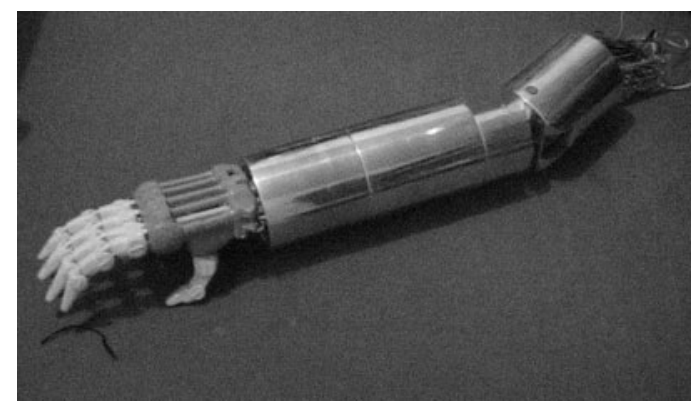

Figure 1 Prosthetic elbow.

experimentally on the biceps. The grasp could be generated through other muscles of the body.

A complete statistical analysis of the EMG signals corresponding to the above motions was first reported by Saridis and Newman (1978), who used simulated amputation measurements as described in the next section. The results indicated that the generated signals - owing to their nonstationary properties - are unreliable for time series analysis and physiological interpretation, but their true averages may serve as feature vectors for pattern classification.

Subsequently, statistical pattern recognition algorithms were used to classify the signals to classes corresponding to each of the possible signal and combined primitive motions of the arm, and their misclassification error was studied. Loading and velocity conditions found to be linearly related to the latter's relations to the features associated with the classification problem, for example, zero crossing (ZC) and signal variance, were established (Bigland and Lippold 1954). Using envelopes of the classes for all loads and velocities, a classification algorithm independent of varying loading and velocity conditions was obtained. Finally, by discovering certain linear superposition properties on the statistics of the features, it was possible to decompose combined motion to their relative velocities.

Load information was obtained through the integral of the muscular activity variable evaluated from the $\mathrm{ZC}$ and variance measurements. The generation of the control algorithms, as well as further elaboration on the muscular activity variable, will be reported in a follow-up paper aimed at the direct control of a prosthetic arm by EMG signals with minimum effort or training on the part of the amputee.

\section{EXPERIMENTAL PROCEDURE}

The EMG signals are easily gathered using skin electrodes. In this study, two electrode sites were used. At each site, one differential silver-silver chloride electrode is separated by a ground electrode. They are separated from the skin by gel-impregnated foam, and were attached by an adhesive foam pad. The electrodes are placed parallel to the humeral axis, slightly off the center of the bulge of each of the biceps. The locations are the lowestmost placements that are undamaged in most above-the-elbow amputees for whom this work will be applicable (Newman and Saridis 1978; Rimroth et al. 1978; Saridis and Newman 1979; Wirta

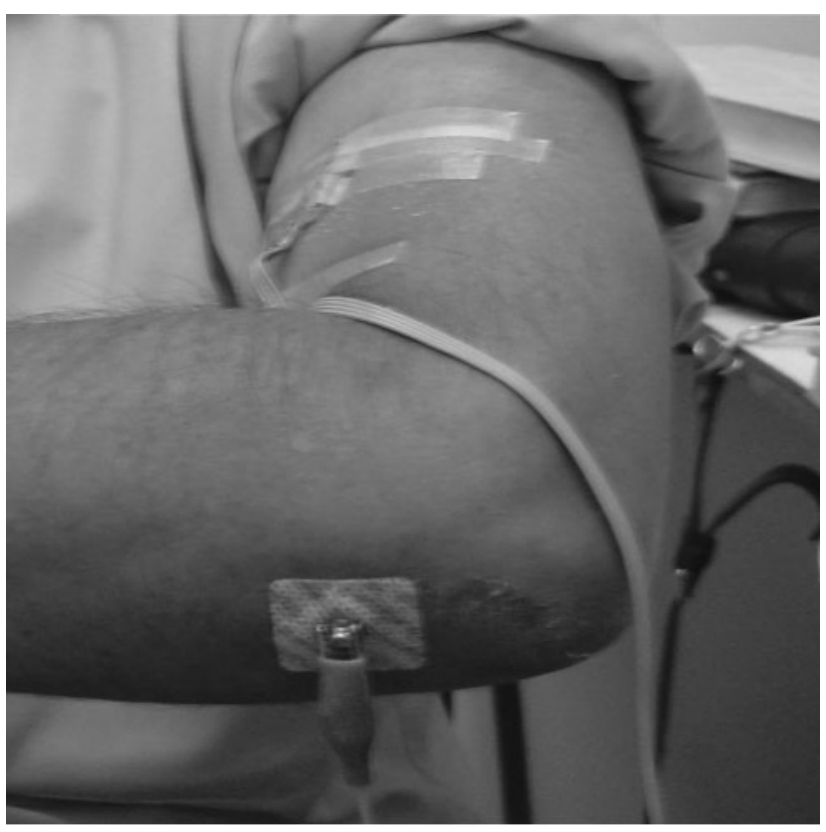

Figure 2 Electrode. Test on real muscle.

et al. 1968). An on-board microcomputer should sample the two signals and sequentially update its decision as to which motion is meant to be in progress. The stream of decisions is fed into a coordinator of the motions of the arm. This is done at intervals of several milliseconds. The amputee's visual feedback of the arm's position and velocity should thus provide accurate enough correction of the motion. We note that the coordinator is designed with a memory to provide trend characteristics of the decision stream to eliminate inadvertent errors and improve classification accuracy.

Experimental data were obtained from an amputation simulator instead of a real amputee, where the subject would completely immobilise his arm in a cast at and below the humerus. The device electrode is shown in Figure 2 (Saridis and Newman 1979). The reason for the simulation was, first, availability of more subjects from a student population, for example, three different subjects have already been used, and second, the flexibility to use the results for designing either upper limb prostheses or orthoses. The results are claimed to be representative of EMG signals from actual amputees with nonatrophised musculature because with the cast, the subject would generate the same synergistic signals over his biceps when he would attempt to move his immobilised arm.

The experiments were performed with the arm in a vertical position. Small-angle variations of the arm position around the perpendicular tested showed no effect in the classification. Over $30^{\circ}$ angle changes in the position of the arm from the vertical, as well as a test on real amputees, are planned in a later phase of this project.

The amputation simulator is constructed of cast aluminum and extends from just proximal to the elbow to the fingertips. The cast holds the elbow at a mid-elbow flexion of $90^{\circ}$, humerus to forearm, and pronation-supination. 


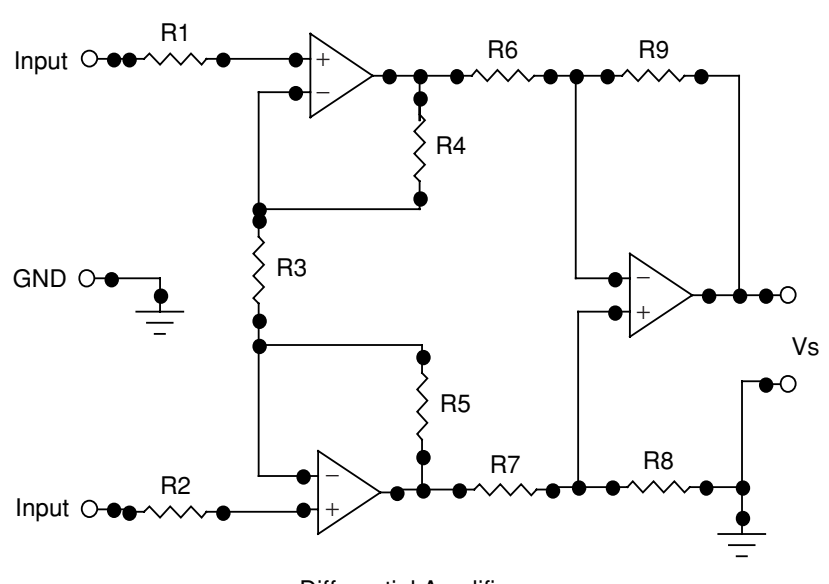

Differential Amplifier

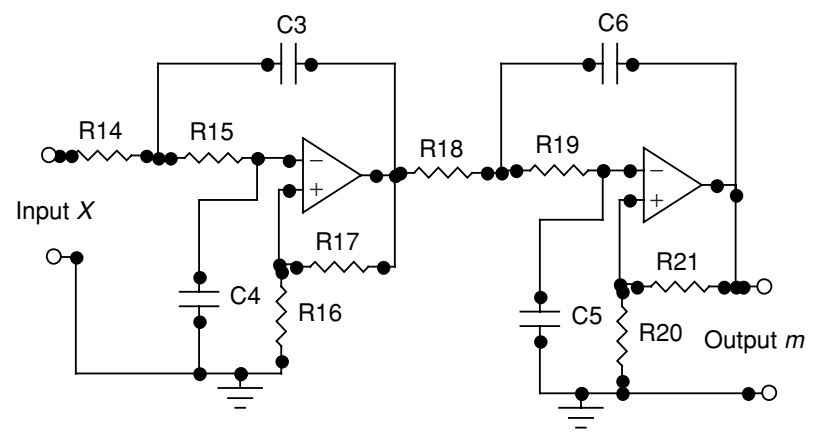

Low-pass filter

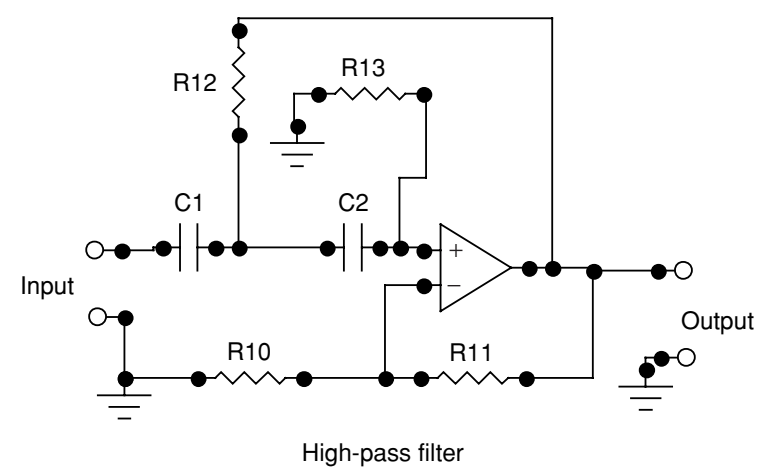

Figure 3 EMG amplifier and filters.

When the shoulder has normal freedom of motion, its position may be monitored through potentiometers mounted at each pin joint. The aluminium elbow mounting bracket for the two high-gain, high input impedance EMG amplifiers is shown in Figure 3.

Twenty-nine motions were defined, along with one control (zero) motion, each consisting of either grasp open/close, or one to three of the wrist, elbow and humeral primitives. A more detailed description is given in Newman and Saridis (1978) and Saridis and Newman (1979). Since the biceps waveforms apparently contained very little information from the grasp primitives, classification of the grasp primitives was not pursued further in this study.

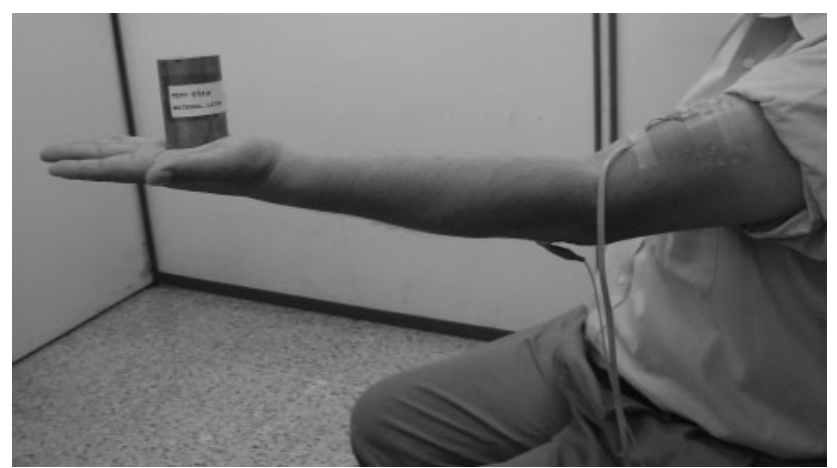

Figure 4 Outline of the arm with the weight, showing the vectors that intervene in the taking of the signals.

\section{STATISTICAL EMG ANALYSIS}

These signals will be registered in a team cyberamp 100 , with gain (100), for conditioning of the signals, with joining of AC of $0.01 \mathrm{~Hz}$ using another cyberamp 380, with gain (50), with joining AC of $10 \mathrm{~Hz}$. After filtration it passes the first floor of $400 \mathrm{~Hz}$. We also have a filter that eliminates the noise generated in the band of $60 \mathrm{~Hz}$.

A card digidata $1322 \mathrm{~A}$ with 16 resolution bites occupies a frequency of sampling of 2000 per second. At first a weight is placed in the palm of a person for $5 \mathrm{~s}$ in a seated position, and this is repeated with different weights (Figure 4). The weights used are as follows:

\begin{tabular}{lc}
\hline Weights & \\
\hline P1 & $240 \mathrm{~g}$ \\
P2 & $610 \mathrm{~g}$ \\
P3 & $650 \mathrm{~g}$ \\
P4 & $940 \mathrm{~g}$ \\
P5 & $1.74 \mathrm{~kg}$ \\
P6 & $2.98 \mathrm{~kg}$ \\
P7 & $3.24 \mathrm{~kg}$ \\
\hline
\end{tabular}

Sequences of EMG data were obtained with a student of normal constitution, 27 years old, making two series, one in the morning and the other in the afternoon.

\section{Morning}

\begin{tabular}{lc}
\hline Name of the file: Sitting position & Event \\
\hline EMG19_0000 & P5+P6+P7 \\
EMG19_0001 & $\mathrm{P} 5$ \\
EMG19_0002 & $\mathrm{P} 5+\mathrm{P} 1$ \\
EMG19_0003 & $\mathrm{P} 5+\mathrm{P} 1+\mathrm{P} 2$ \\
EMG19_0004 & $\mathrm{P} 5+\mathrm{P} 1+\mathrm{P} 2+\mathrm{P} 3$ \\
EMG19_0005 & $\mathrm{P} 5+\mathrm{P} 1+\mathrm{P} 2+\mathrm{P} 3+\mathrm{P} 4$ \\
EMG19_0006 & $\mathrm{P} 5+\mathrm{P} 6$ \\
EMG19_0007 & $\mathrm{P} 5+\mathrm{P} 7$ \\
\hline
\end{tabular}




\begin{tabular}{lc}
\hline Name of the file: Foot position & Event \\
\hline EMG19P_0000 & P5+P6+P7 \\
EMG19P_0001 & P5 \\
EMG19P_0002 & P5+P1 \\
EMG19P_0003 & P5+P1+P2 \\
EMG19P_0004 & P5+P1+P2+P3 \\
EMG19P_0005 & P5+P1+P2+P3+P4 \\
EMG19P_0006 & P5+P6 \\
EMG19P_0007 & P5+P7 \\
\hline
\end{tabular}

\section{Afternoon}

\begin{tabular}{lc}
\hline Name of the file: Sitting position & Event \\
\hline EMG19V_0000 & P5+P6+P7 \\
EMG19V_0001 & P5 \\
EMG19V_0002 & P5+P1 \\
EMG19V_0003 & P5+P1+P2 \\
EMG19V_0004 & P5+P1+P2+P3 \\
EMG19V_0005 & P5+P1+P2+P3+P4 \\
EMG19V_0006 & P5+P6 \\
EMG19V_0007 & P5+P7 \\
\hline
\end{tabular}

\begin{tabular}{lc}
\hline Name of the file: Foot position & Event \\
\hline EMG19VP_0000 & P5+P6+P7 \\
EMG19VP_0001 & P5 \\
EMG19VP_0002 & P5+P1 \\
EMG19VP_0003 & P5+P1+P2 \\
EMG19VP_0004 & P5+P1+P2+P3 \\
EMG19VP_0005 & P5+P1+P2+P3+P4 \\
EMG19VP_0006 & $\mathrm{P} 5+\mathrm{P} 6$ \\
EMG19VP_0007 & $\mathrm{P} 5+\mathrm{P} 7$ \\
\hline
\end{tabular}

These registrations were introduced into a program, to be run in the MatLab software, to obtain the corresponding graphs for each one of the cases, some of which are shown here.

Once the data are registered, MatLab provides the mean values and the securities RMS or the combinations that are wanted.

The mean of the signal, composed of a high number of (more than 500) sample points, is verified by computation in MatLab (Figure 5). The variance $\sigma^{2}$ of the EMG signal $v(t)$ as given by

$$
\sigma^{2}=\frac{1}{800} \sum_{t=1}^{800} v(t)^{2}
$$

It is used as one variable. The absolute value of the moment $\left|\sigma^{3}\right|$ given by

$$
\left|\sigma^{3}\right|=\left|\frac{1}{800} \sum_{t=1}^{800} v(t)^{3}\right|
$$

is another variable. The absolute value is taken to greatly reduce within-class separation. Also computed is the fourth

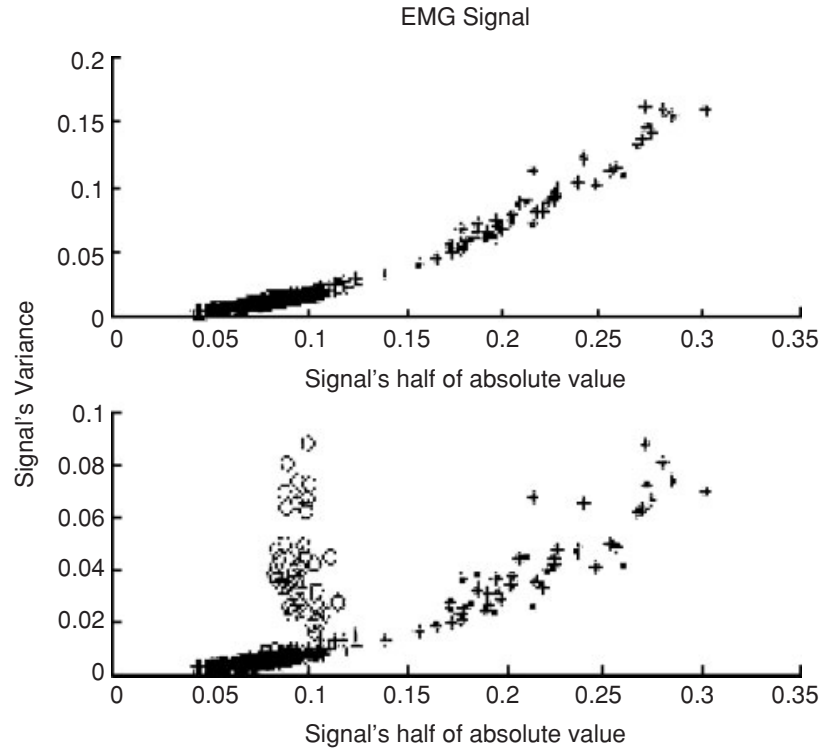

Figure 5 Electromyograph of the variance against the stocking of the absolute value of the signal.

moment $\sigma^{4}$, the absolute value of the fifth moment $\left|\sigma^{5}\right|$, and the number of times the signal potential passes through or touches zero (i.e., ZCs).

Also computed for each signal were the cross correlations between the bicep signal $R_{\mathrm{b}}(\tau)$, the power spectra $S_{\mathrm{b}}(\omega)$, computed by fast Fourier transform, and the signal voltage density $D_{\mathrm{b}}(v)$. Density and correlation were later discarded because they did not apparently contribute class separation, that is, in distinguishing between motions.

$S_{\mathrm{b}}(\omega)$ and $R_{\mathrm{b}}(\tau)$ have both 800 components, each of which is a measurement variable. Although the information content of an autocorrelation and a power spectrum is in a sense redundant, and although $R(0)=\sigma^{2}$, all of these are computed to simplify the process of feature selection. The measurement space, therefore, has 2058 components.

$$
\left[\sigma^{2}+\left|\sigma^{3}\right|+\sigma^{4}+\left|\sigma^{5}\right|+\mathrm{ZC}+R(\tau)+S(\omega)\right]
$$

The final conclusions from the statistical analysis are as follows.

- The EMG signals are not stationary and are band limited to virtually $1200 \mathrm{~Hz}$.

- No synergistic signals are generated from the hand grasp.

- Pattern information of the arm is contained in the ZC and depends on the electrical activity of the muscles.

- Loading and velocity information of the arm is contained in the ZCs and variance, and depends on the internal muscular electrical activity.

- Motion information is concentrated mostly in the variances and less in the higher-order moments.

- In most motions, the variance and ZC information are grouped in separable clusters that may be approximated by Gaussian densities, and their moments represent the classes. 


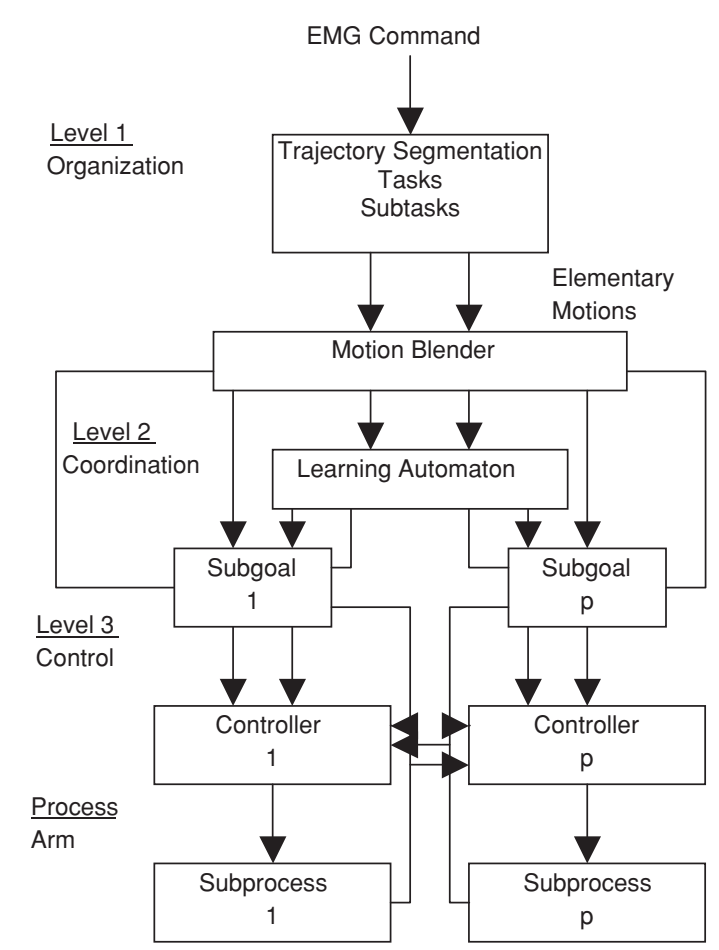

Figure 6 Hierarchically intelligent control of a prosthetic arm.

All of the above indicate that pattern recognition methods may be applicable to classify and recognize the various motions under consideration.

\section{PATTERN CLASSIFICATION FOR EMG SIGNALS}

Hierarchical intelligent control methods (Saridis and Stephenou 1977) may be applicable to drive the prosthetic arm if movement and loading information is property decoded and applied to a microcomputer system, programmed accordingly. Pattern recognition methods are quite adequate to provide such information as will be obvious from this study. The final control configuration is presented in Figure 6 for an arm incremental motion.

In most pattern recognition problems, it is necessary to greatly reduce the measurement space into a feature space of suitable dimension so that the final pattern recognition algorithm can be managed using an on-board microcomputer. This will be accomplished at the end of this study by selecting as features the variables that contain maximum information on class separation.

The variance of the signal was selected as the measure of scatter to determine the importance of each variable. If a variable has large between-class and small within-class scatter, that is, the criterion for class separation, for any two classes, then it is considered important. All unimportant variables are immediately discarded. The ranking of each of the remaining features is determined by the total number of classes separated and the relative amount of separation of the variable. When the feature count can be reduced to 50 or less, a computer can handle the computations necessary for a rigorous mathematical analysis to further minimize the feature vector, while considering any resulting classification error that may be expected (Fukunaga 1972). The learning algorithm (Appendix I) makes it possible to determine the number of iterations for each pair of movements in the elbow.

\section{THE PROPERTY OF SUPERPOSITION OF PRIMITIVES}

Owing to the physical nature of the problem, it seems reasonable, perhaps even more straightforward, to consider only six classes, each defined as the presence of one of the primitives. In doing so, it was noted that using the variances, the primitives exhibit superposition properties, which might provide the means to develop a six-class scheme. That is, linear combinations of the variance data are taken for more than one primitive, the resulting distribution approximates that of the combined motion involving the primitives used. Thus, distributions of combined motions should be decomposed into the distributions of their primitives, possibly permitting primitive identification from the variances of unknown motions.

For each pair of primitive motions, coefficients of a linear combination of points from the two variance distributions were calculated, which resulted in the mean of the derived distribution being equal to the mean of the actual combined motion's distribution (see Appendix II).

\section{CONCLUSIONS}

Considerable data are available regarding communicating information from a below-the-humerus incapacitated subject and a microcomputer system programmed to hierarchically implement intelligent control of a prosthetic arm.

Learning pattern classification methods and primitive decomposition may yield fairly accurate commands on generating effects on this subject for a variety of loading and velocity conditions. Memory of the previous movement and visual feedback may be utilised to interrupt a motion that was incorrectly understood. The features needed are only ZCs and variances, as in simple speech recognition problems (Rabiner and Schafer 1978). The integrated muscular activity may be used to evaluate the load and velocity parameters involved. Further research is aimed at experimental verification of the actual movement and control of the prosthetic arm. The final test of the method will be with actual amputees from the local rehabilitation center.

\section{REFERENCES}

Alderson S. 1954. The electric arm. In Klopsteg PE, Wilson PD, eds. Human Limbs and Their Substitutes. New York: McGraw-Hill, Chap. 13. (reprinted by Hafner, 1969). 
Bigland B, Lippold OCJ. 1954. The relation between force, velocity and integrated electrical activity in human muscles. 7 Physiol, 123:214-24.

Fukunaga K. 1972. Introduction to Statistical Pattern Recognition. New York: Academic Press.

Graupe D, Salahi J. 1979. Multifunctional Artificial Limb Control via EMG Temporal Analysis-Background and Amputee Tests [PhD thesis]. Department of Electrical Engineering, Illinois Institute of Technology, Chicago.

Graupe D, Magnussen J, Bees A. 1978. Microprocessor system for multifunctional control of upper-limb prostheses. IEEE Trans Automat Contr, AC-23:538-44.

Lyman J, Freedy A, Zadaca H. 1974. Studies and development of heuristic end-point control for artificial upper limbs. UCLA Biotechnology Laboratory. Technical report 54.
Newman MA, Saridis GN. 1978. Development of prosthetic arm. Purdue University, West Lafayette, IN. AARL Memo 30.

Rabiner LR, Schafer RN. 1978. Digital Processing of Speech Signals. Englewood Cliffs, NJ: Prentice-Hall.

Rimroth P, Newman MA, Saridis GN. 1978. A study of electromyography for above-elbow amputation. Purdue University, West Lafayette, IN. AARL Memo 34.

Saridis GN, Newman MA. 1979. Upper limb EMG statistical analysis. In Proceedings of MIDCON'79, Chicago, IL.

Saridis GN, Stephenou HE. 1977. A hierarchical approach to the control of a prosthetic arm. IEEE Trans Syst Man Cybernet, SMC-7:407-20.

Wirta RW, Taylor DR, Finley FR. 1968. Engineering principles in the control of external power by myoelectric signals. Arch Phys Med, 49:294-6. 
APPENDIX I: THE STOCHASTIC APPROXIMATION ALGORITHM FOR COMPUTATION OF DISCRIMINANT COEFFICIENTS

The general linear-discriminant classifier for two classes $\omega_{1}$ and $\omega_{2}, V$ can be written as

$$
h=V^{T} X+v_{0} \begin{gathered}
> \\
<
\end{gathered} 0 \Rightarrow X \in\left\{\begin{array}{l}
\omega_{1} \\
\omega_{2}
\end{array} .\right.
$$

This can be rewritten as

$$
\begin{aligned}
& h=W^{T} Y_{<}^{>} 0 \\
& h=\sum_{i=0}^{n} w_{i} y_{i},
\end{aligned}
$$

where $y_{0} \equiv 1, y_{1}=x_{1}$, that is, $w_{0}=v_{0}$.

Now, if we let $Z=Y$ for $Y \in \omega_{1}$, and $Z=-Y$ for $Y \in$ $\omega_{2}$, then $W^{T} Z>0$ for correct classifications.

The stochastic approximation can be expressed as

$$
W_{l+1}=W_{l}-a_{l}\left[W_{l}^{T} Z_{1}-\gamma\left(Z_{l}\right)\right] Z_{l}
$$

where $\gamma\left(Z_{1}\right)$ is the desired classifier output $(>0)$ for $Z_{1}$, and $a_{1}$ is required to satisfy

$$
\lim _{l \rightarrow \infty} a_{1}=0, \sum_{l=1}^{\infty} a_{1}=\infty, \text { and } \sum_{l=1}^{\infty} a_{l}^{2}<\infty
$$

To guarantee convergence, for example, $a_{1}=1 / l$. In order to speed up the convergence, the number $a_{1}=1 / k$ was advanced to $1 / k+1$ only when the term $\left[W_{l}^{T} Z_{l}-\gamma\left(Z_{1}\right)\right]$ changed sign.
APPENDIX II: SUPERPOSITION OF PRIMITIVES TO MATCH THE MEAN OF MULTIPLE PRIMITIVE MOTIONS

Let the mean of the double-primitive motion being considered be

$$
\left[\frac{\sigma_{B}^{2}}{\sigma_{T}^{2}}\right]
$$

Let the data of the component primitives be

$$
\left[\begin{array}{c}
\sigma_{B i k}^{2} \\
\sigma_{T i k}^{2}
\end{array}\right] \text { and }\left[\begin{array}{c}
\sigma_{B j k} \\
\sigma_{T j k}^{2}
\end{array}\right], \quad i=1,27, j=1,20 .
$$

The data are used with the equation

$$
\left[\begin{array}{c}
\sigma_{B \text { new }}^{2} \\
\sigma_{T \text { new }}^{2}
\end{array}\right]=c_{1} \cdot\left[\begin{array}{c}
\sigma_{B i k}^{2} \\
\sigma_{T i k}^{2}
\end{array}\right]+c_{2} \cdot\left[\begin{array}{c}
\sigma_{B j k}^{2} \\
\sigma_{T j k}^{2}
\end{array}\right]
$$

with coefficients $c_{1}$ and $c_{2}$ such that the mean of the $\left(\sigma_{B \text { new }}^{2}, \sigma_{\text {Tnew }}^{2}\right)$ distribution is equal to

$$
\left[\frac{\sigma_{B}^{2}}{\sigma_{T}^{2}}\right]
$$

$c_{1}$ and $c_{2}$ are easily found by

$$
c_{1} \bar{\sigma}_{B i}^{2}+c_{2} \bar{\sigma}_{T j}^{2}=2 \bar{\sigma}_{B}^{2} \text {. }
$$

And

$$
c_{1} \bar{\sigma}_{T i}^{2}+c_{2} \bar{\sigma}_{T j}^{2}=2 \bar{\sigma}_{T}^{2} .
$$

The solution of (AII.1) and (AII.2) is

$$
\begin{aligned}
& c_{2}=\frac{\left(2 \bar{\sigma}_{T}^{2}-2 \bar{\sigma}_{B}^{2} \bar{\sigma}_{T i}^{2} / \bar{\sigma}_{B i}^{2}\right)}{\left(\bar{\sigma}_{T j}^{2}-\bar{\sigma}_{B j}^{2} \bar{\sigma}_{T i}^{2} / \bar{\sigma}_{B i}^{2}\right)} \\
& c_{1}=\frac{\left(2 \bar{\sigma}_{B}^{2}-c_{2} \bar{\sigma}_{B i}^{2}\right)}{\bar{\sigma}_{B i}^{2}} .
\end{aligned}
$$



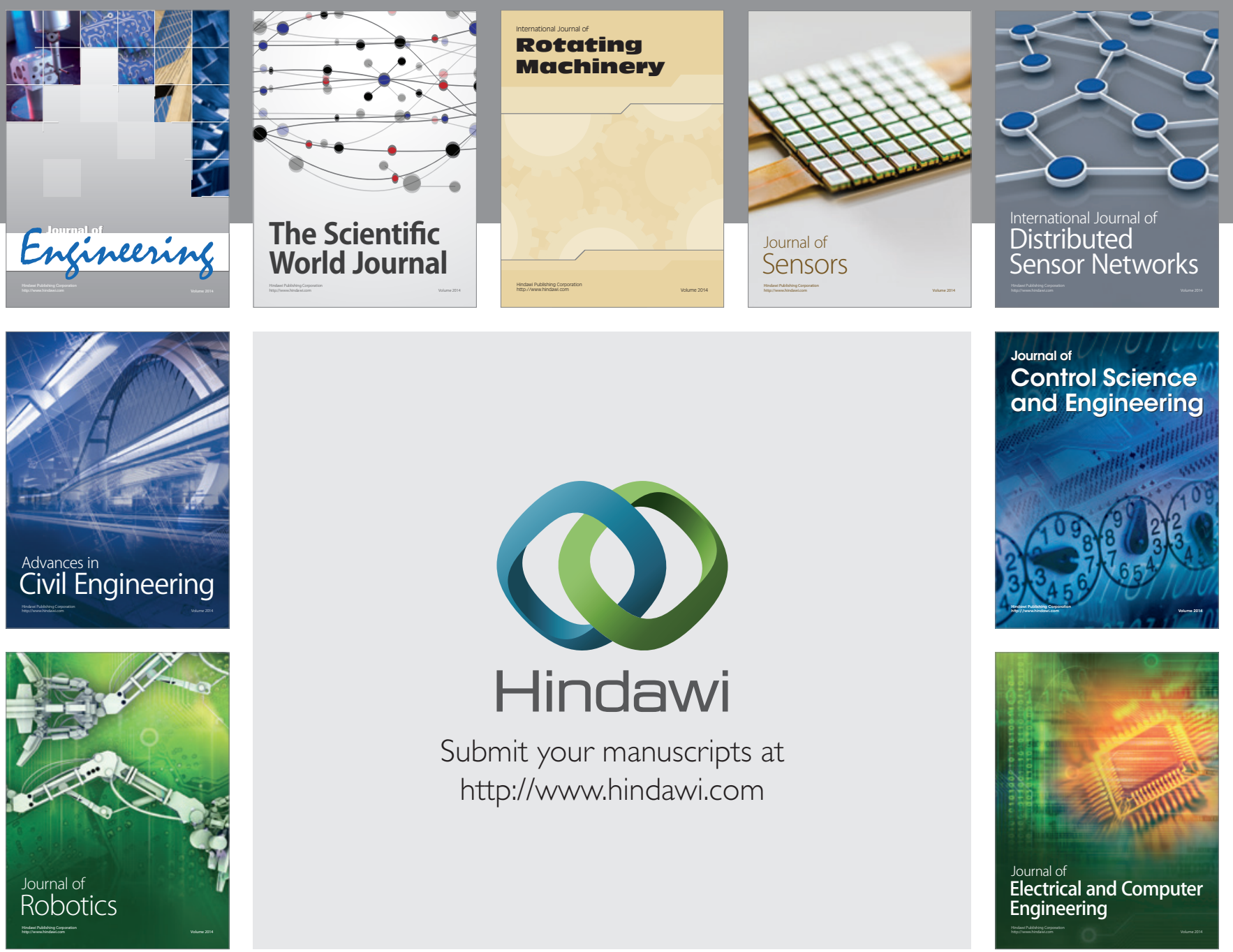

Submit your manuscripts at

http://www.hindawi.com
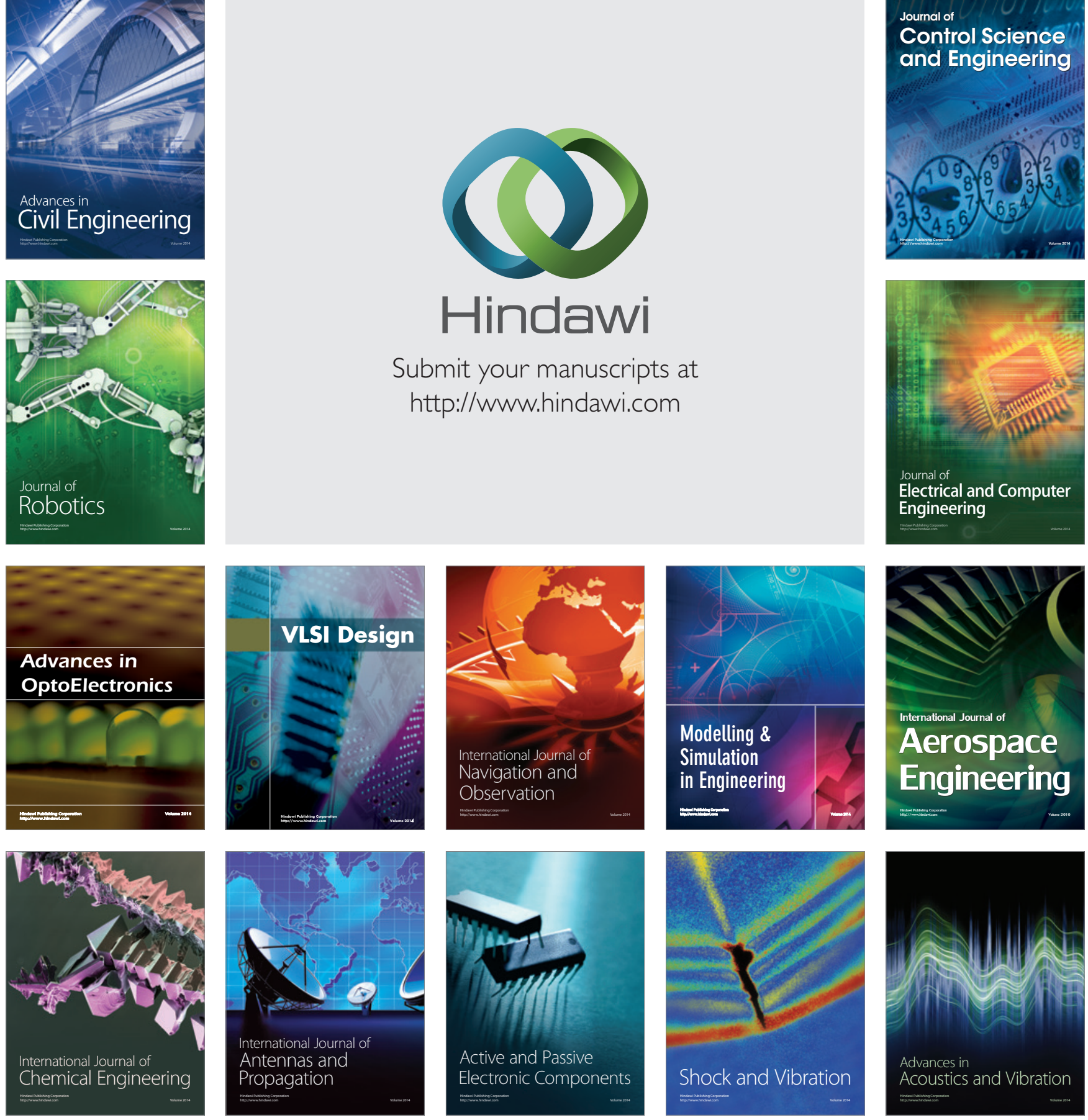